\title{
Semiotic-based Conceptual Modelling of Hypermedia
}

\author{
Elio Toppano and Vito Roberto \\ Department of Informatics, University of Udine, Italy \\ \{elio.toppano, vito.roberto\}@uniud.it
}

\begin{abstract}
We address the conceptual modelling of hypermedia regarded as semiotic texts, whose meanings are conceived by a designer, transferred through the artifact and interpreted by users within their context. We outline the communication framework, with the artifact embedding the images of the designer and user. The full model is represented in terms of four interrelated modules: story, discourse, text and social-relational ontologies. The first and second one account for the narrative structures underlying the hypermedia content, which is externalized through sensorial qualities that, in turn, evoke impressions into the user. The model addresses issues that are poorly covered by the description standard MPEG-7. It can be used for analysis, evaluation, indexing of existing hypermedia as well as the design of new ones.
\end{abstract}

Keywords: Modelling, hypermedia, ontologies, semiotics.

\section{Introduction}

In the field of hypermedia design, a renewed interest is being due to the construction and transfer of meaning, as well as to the study of interpretive processes underlying the user media experience [1], [2]. Meanings are the result of a design, intended for a class of users inscribed into the product. The latter is a mediator towards the user who reconstructs (i.e., interprets) the meaning according to his/her goals and contexts [3], [4]. Such an approach is more comprehensive (holistic) as compared with the design models inspired to software engineering methodologies [5], [6], and promising towards a semantically-oriented modelling of hypermedia which is the subject matter of the present paper. We propose a set of ontologies and a vocabulary, adopting semiotic concepts. Earlier attempts to use Semiotics concern the design of multimedia interfaces, the so-called Semiotic Engineering [7]; a semiotic model of information artifacts is reported in [8]. A more complex model [9] was inspired to the generative construction of meaning. A number of reasons motivate the choice of Semiotics in the present context. First, the fact that semantics is strictly related to a dynamic, participative construction of meaning by designers and users appears a reasonable assumption. Second, the concept of text - intended in a general way as a system of related signs - provides a scaffolding to integrate the multiple forms of hypermedia. It also provides a solution to the semantic gap problem, i.e., the difficulty to assign semantics to the low-level features due to the lack of ontological bridges among concepts from different conceptualizations. Third, the idea of media experience is strictly related to that of narrative, i.e., a primary subject of interest of a semiotic investigation. Last, a number of social relationships are

A. Petrosino (Ed.): ICIAP 2013, Part II, LNCS 8157, pp. 663-672, 2013.

(C) Springer-Verlag Berlin Heidelberg 2013 
involved and accounted for, along with the cultural issues - the distribution of shared values and points of view within a social context. The paper is organized as follows. A critical discussion about the MPEG-7 content description gives us the opportunity to introduce the basics of our meta-model, which is presented in Sections 2 and 3. Section 4 contains the ontologies that detail the model under different perspectives. Our conclusions are reported in Section 5.

\section{The MPEG-7 Content Description}

It is the metadata initiative that aims to create a standard content description which also deals with the meaning of information [11], [12], [13]. It includes four components: the Description Definition Language (DDL); the Audio; Visual and Multimedia Description Schemes (DS). MPEG-7 does not incorporate formal semantics and is based on XML-encoded metadata. Although it is not a meta-model like AHM [5], it embeds a conceptual model, i.e., an abstraction of the artifacts that can be described by the standard.

Content Entities. MPEG-7 does not apply a clear-cut distinction among the data, their physical manifestation and their meanings. Actually, it is not clear what the concepts Multimedia Content and Content Entity refer to in [10], [14]. It appears that the content structure refers to the organization of both the media source and the spatial-temporal layouts of the rendered data. However, those structures refer to distinct "structuring spaces": the former concerns the ways data are packaged within the format container e.g., a file; the latter, the organization of visual/aural signs within the presentation space - e.g., a display. The need is apparent of a distinction on conceptual grounds. A step forward is accomplished by addressing semiotic processes. A digital object - the media source - is a sign in the real world to be interpreted by the HW/SW platform in terms of another sign, presented through the interface. The latter signs, organized into a text, act as stimuli for further semiotic processes involving the user.

The Narrative Perspective. MPEG-7 adopts a narrative perspective. Accordingly, the Authors distinguish among reality, content entities and narrative worlds, which is consistent with the view of the early semiotician C.S. Peirce [15]. However, the perspective does not seem to have been consistently maintained by the Authors. Although the Descriptor Schemes provided by the standard - object DS, event DS, concept DS, - and the normative semantic relations - agent, patient, beneficiary, - are, in principle, general enough to represent narrative structures, the analyst is left alone in this task. The need is apparent of aggregated concepts to represent narrative programs or schemas, such as the canonical one by A.J. Greimas [16]. We propose to adopt a true narrative stance; we articulate the model into three levels: story, discourse and text [17], and provide a specific ontology for each. The choice to regard the hypermedia as a narrative text is independent from the particular genre, like storytelling. We claim that narrative naturally unfolds because of the receiver's attempts to make sense, no matter whether the product actually tells a story. 
Creation Information Tools. The tools describe the generation process of a content entity by explicitly assuming that hypermedia have little or no relation with the stakeholders designers, users, ... - responsible of their ideation, development and use. Therefore, content is reduced to what its media components - text, video, audio, ...- represent. With a different stance, we claim that a content entity is always the result of a design, which inevitably embodies the imprinting (images) of both the authors and the intended users. Our proposal is to explicitly account for the images of sender and receiver as inscribed within the hypermedia. This is relevant in application areas like brand marketing and edutainment, where the quality of a product is assessed by looking not only at its technical features, but also at the effectiveness in communicating the brand identity, or the author intentions to teach, persuade, inspire.

Sensorial Features. The standard proposes a set of tools that describe visual and audio features. The former include color, texture, shape and motion descriptors; the audio, descriptors for time and spectral analysis of signals. The tools are useful for content retrieval, genre classification, mood identification, content matching; they allow to represent features of a hypermedia at a physical and perceptual levels. The need arises to relate the features to higher perceptual and cognitive impressions. We explicitly link sensorial qualities to abstract features such as the aesthetic, affective, and ludic impressions that can be evoked [4]. As a consequence, the way is open to represent the intended user's experience and exploit this information for "experience-based" retrieval.

The Active User. The standard provides descriptors for the semantic annotation of narrative worlds as depicted in multimedia content. The potential events involving the user are not taken into account. Therefore, the standard does not support interactive products in which the user takes an active role in the story - e.g., she is a character of the story - or selects the next scene to be performed. We shall detail both roles in section 4.2. As a matter of fact, sender and intended user are not explicitly represented, so it is not possible to assign them some action/event in the story nor in the discourse. Our proposal is to account for a possible world, by which we mean the set of potential events - narrative programs - inscribed within the hypermedia, including those associated to the image of the sender (addresser) and that of the user (addressee), as sketched in Figure 1 and 2 .

\section{Hypermedia as Semiotic Objects}

Figure 1 shows our communication framework. The real sender and receiver are connected by means of an instrumental medium (vehiculum)- such as a PC connected to the Net - which enables the circulation of the hypermedia. The sender is usually a team of designers or multimedia developers, sponsors and stakeholders. The interactions between sender and hypermedia take place within a production context, are iterative and incremental including reflections and talkbacks. The receiver, an individual or a group, interacts with the product within a context of use. Sender and receiver communicate through the hypermedia, whose meaning undergoes three stages of development. 
At first, the intended meaning is defined, and included into the product at the second stage (inscribed meaning). The receiver interprets the artefact as an object in her/his mind (reconstructed meaning). The same features conceived by the designer act as stimuli and constraints that evoke the intended meanings into the user. The content of a design is not merely the artifact itself, but triggers a flow of consciousness into the receiver (an experience), that is constructed through the receivers fruition of the product.

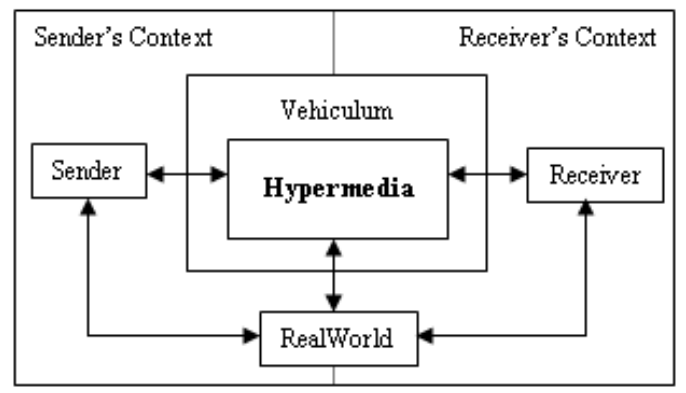

Fig. 1. A communication-based design framework. The central hypermedia box is further expanded in Figure 2

The hypermedia is regarded as a structured whole of signs also called a text - like written words, images, sounds, ... - representing a narrative repackaging (discourse) of the set of virtual courses of events (story). The narrative describes potential interactions (possible world) occurring between sender and receiver. Interactions are embodied/inscribed within the text in a frozen state and reactivated (i.e., enunciated) during use. The reflected images (simulacra) of sender and receiver are the addresser and addressee, respectively (see Figure 2). The former is represented, for example, by visual logos. Analogously, the addressee is implicitly represented by the design choices to be shared by the user, such as lexicon or accessibility issues. Addresser and addressee may be different from the real sender and receiver, respectively. Discourse and story define a possible world.

\section{An Ontology}

The ontology we propose has been organized into related modules: the story and discourse; agent; text; and social relational ontologies. Figure 2 (right) shows an overview of modules and relationships.

\subsection{Story and Discourse Ontologies}

The term 'story' denotes what is depicted in a narrative while the discourse is about how it is told [17]. In some narratives story and discourse - as defined in Sec. 3 match simply, while in other they are markedly different. For example, the sequence 

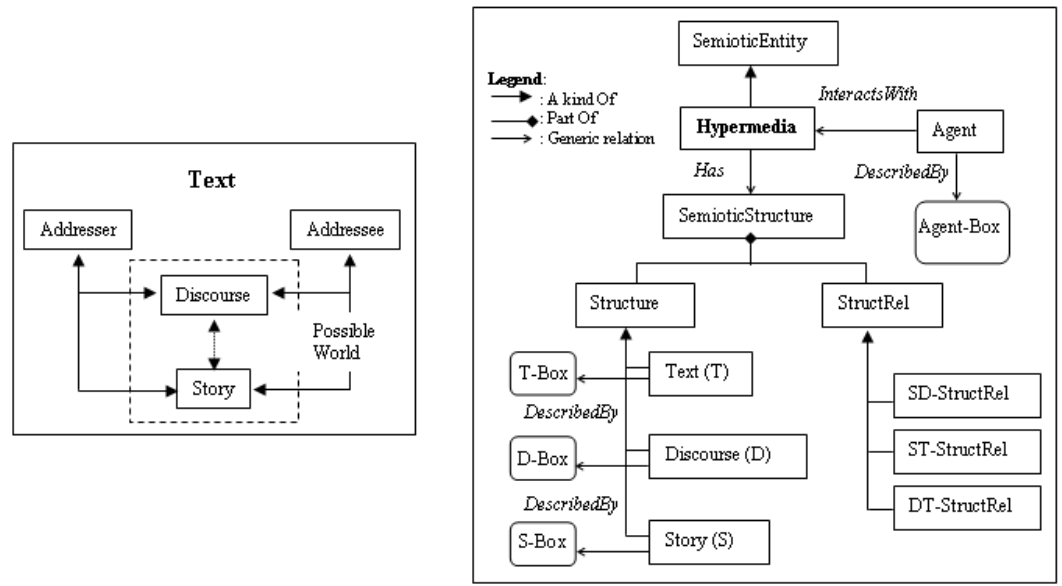

Fig. 2. Left: inside the hypermedia box in Figure 1 Right: the hypermedia conceived as a semiotic text.

of story events may be modified by acting on the time order, duration and frequency, thus producing discourse effects like flashbacks. Due to space constraints and similarity of conceptualizations, we focus on the discourse model (Figure 3). It has a structure (D-Structure) composed by segments (D-Segment) and relations (D-SegmentRel). Each segment is viewed as a combination of a D-Setting and a D-NarrativeStructure. The former specifies place, time and a mise-en-scene. The D-NarrativeStructure describes the activities occurring on the setting in terms of D-NarrativePrograms. In turn, a narrative program is specified by a D-Agent performing a D-Action and causing a D-Effect. Actions include processes that have been classified according to [18]. Narrative programs and segments are inter-related by logical, temporal or rhetorical links. Transformations between discourse segments occur whenever one of their constituent elements - i.e., a setting, a narrative structure undergoes a change.

\subsection{The Agent Ontology}

Addresser and addressee are agents playing multiple roles both in story and discourse, as described in Figure 4 . Agents of a story (S-Character) represent abstract characters, or roles in a plot. Agents in a discourse (D-agents) are real actors interpreting characters in a specific realization of the plot. They may be human or artificial, like an avatar in a digital game. Addresser and addressee play the role of other D-agents: i) subjects of perception; ii) subjects of montage or iii) subjects of production. Subject of perception is responsible for focalization of discourse: physical focus is expressed by a specific choice of shot types, camera angles, lighting. Cognitive, affective and ideological focalizations concern values, points of view, feelings the designer intends to communicate. Analogously, the subject of montage and production are agents responsible of composing and realizing discourse segments, respectively. 


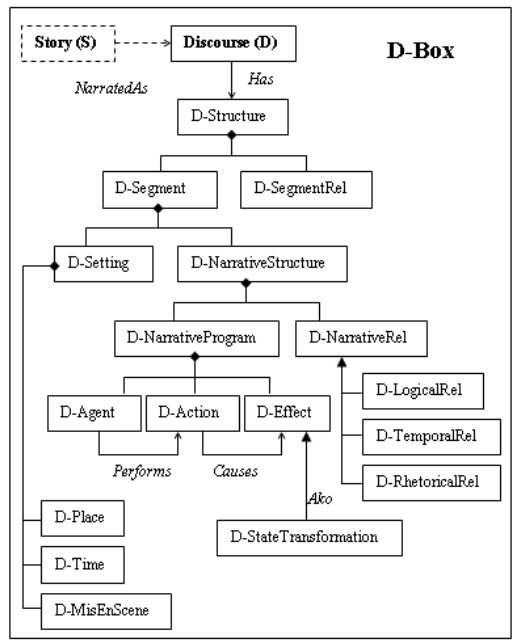

Fig. 3. The Discourse ontology, the D-Box in Figure 2

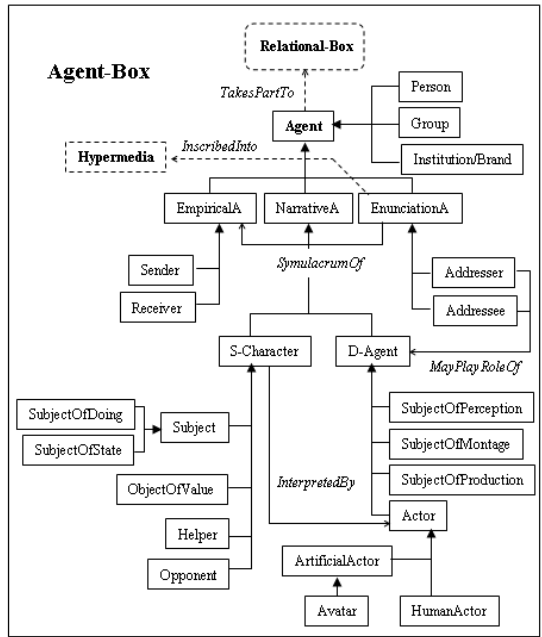

Fig. 4. The Agent ontology

\subsection{The Text Ontology}

Text is the concrete manifestation of the discourse. It deals with signs, elements that convey narrative content to the user interpretation. This ontology plays a key role within the conceptualization as a whole, since the fundamental concepts of content and expression belong to the text structure, as shown in Figure 5. On the content side, a text segment (T-Segment) is linked to the associated discourse chunk (D-Segment), which in turn is related (Figure 3) to a setting, a narrative program and a story segment. 


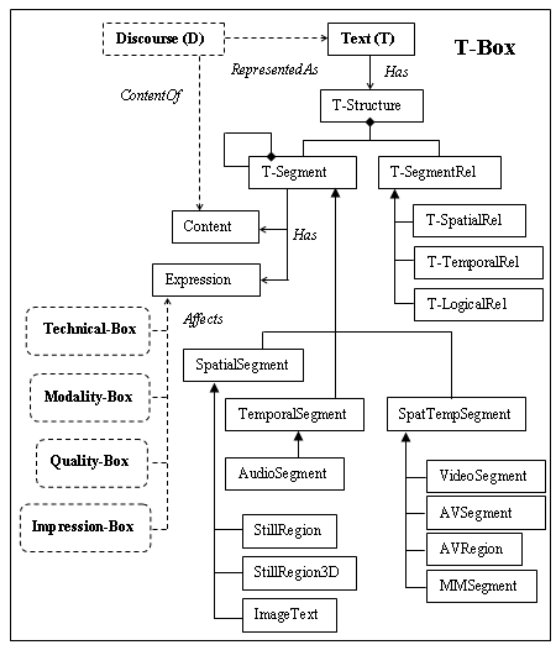

Fig. 5. The Text ontology (the T-Box in Figure 2). Text segments have been classified according to the MPEG-7 standard.

On the other side, the T-segment points to four lower-level substructures which together conceptualize the expression: we list them in the sequel.

TechnicalBox: expression is influenced by technical features of the HW/SW platform and digital objects. The latter are sources of data (e.g., a file), rendered by a T-segment. As an instance, a JPEG file is rendered as a still fragment depicting an image on the screen. The link between digital object and its rendering is realized by a HW/SW platform - a computer, a mobile phone, ...- containing tools to reproduce content. This ontology has not been shown.

ModalityBox: expression is related to the modality of content representation: image, written and spoken language, music, animation, audiovisual, sound and video effects.

QualityBox: expression has sensorial qualities and quality relations. We distinguish between tonal static, persistent and rhythmic dynamic, transient qualities. Sensory qualities are inter-related by contrast, completion, affinity or analogy, according to the structural relations existing among their associated segments (see Figure 6).

ImpressionBox: the sensorial qualities of expression underlie interpretive activities and impressions affecting the users experience.

\subsection{The Social Relational Ontology}

Relations between sender and receiver arise through their images within the text. An interesting view in [19] holds that the designer actually creates a persuasive argument that comes to life whenever a user considers the product. A hypermedia can be shaped by persuasive intentions: generating attraction towards the product; fostering the attribution of meanings; promoting self-identification with the product; stimulating positive responses like satisfaction and delight. Intentions may be inferred from the product and 


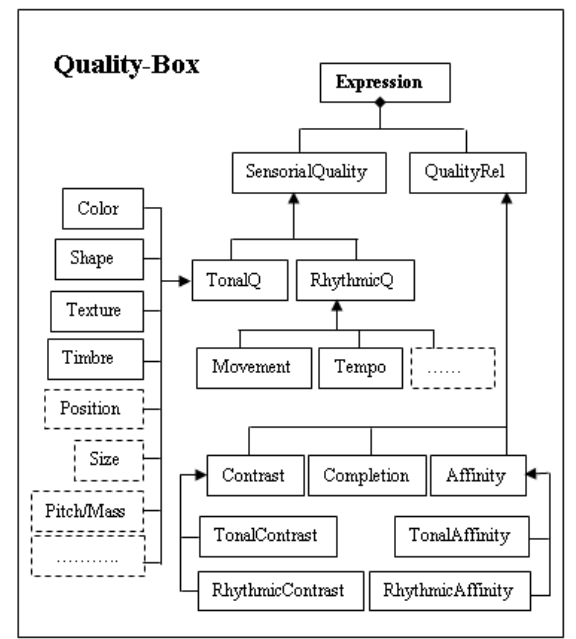

Fig. 6. The exploded description of the QualityBox in Figure 5

possibly shape the receivers experience. We guess that persuasion is primarily achieved by aesthetic, affective and ludic impressions evoked by the text. A kernel ontology for impressions includes:

Pragmatic impressions: are related to functional qualities like utility, efficacy, security.

Aesthetic pleasure: is intended here as "sensorial delight" [20] and refers to product appearance and interaction. As an instance, the combination of visual tonal qualities affects the aesthetics of visual appearance through proportion, equilibrium, symmetry [21].

Enjoyment: the experience of fun and pleasure as discussed in [22]. Fun is related to intensity of perceptual stimuli; pleasure, with quality of perception.

Emotion: a kind of affective phenomenon. According to the Appraisal Theory [23] emotions arise in response to the appraisal of a stimulus event as relevant to major concerns of the organism. Emotions can be represented by the independent dimensions of arousal and valence [24]. Since also sensorial qualities can be mapped into the same space, it is possible to bridge the gap between sensorial experience and some affective phenomena.

Immersion: a measure of how persuasive an environment is and how persuaded are the users. According to [25] we distinguish between flow and presence. Flow arises from immersion in a task; presence, from immersion in an environment.

Identity: captures impressions related to user self-expression and self-worth - e.g., identity and lifestyle.

A core ontology of social relations focuses on empathy and trust. By the former we mean the process through which the receiver enters in relationship with another subject and possibly share her/his sensorial, perceptive, cognitive or affective status. We indicate the subject and object of empathy as the empathiser and empathee, respectively [26]. Trust means "trusting an agent", i.e., its ability to suggest effective actions; 
show beautiful objects; tell the truth. According to the Agent ontology in Figure 4 the receiver represented by the addressee - can enter in relational empathy with two agents inscribed within the text, the subjects of story and discourse. Empathy with the latter is crucial for credibility of a discourse, its aesthetic value and technical quality. All of these issues affect the user's trust.

\section{Conclusions}

We have proposed a conceptual model inspired to the Semiotic theories of Peirce and Greimas. Hypermedia are objects purposively designed to transfer values and achieve communication goals. Their meanings arise from dynamic processes of semiosis, and result from design choices, mediating role of the object, interpretation of a user. Within a holistic scheme, designers and users are regarded as interlocutors by means of the document, which contains their images and enables dialogue within a possible world. Our model consists of three conceptual spaces text, discourse, story interconnected by relations which outline a structured picture of semantics. Our approach results in a number of ontologies that are original contributions of the present paper. Ontologies provide a conceptual, systematic view of the hypermedia under multiple perspectives. On the designer side, the hypermedia is described under three design spaces, with specific vocabularies, linked by suitable ontological bridges. On the user side, the model enables to detail the media experience with a product in terms of sensorial, narrative and relational points of view. We believe that, ultimately, our meta-model addresses the hypermedia as the result of an experience-oriented design, and is an attempt to overcome the limitations of current product-oriented and user-oriented approaches. The conceptual framework we propose can be used for analysis, evaluation and annotation of existing hypermedia, as well as the design of new products. The vocabulary enables designers to fully express their intentions to address the user experience. Tools are given to teach the "experience literacy" similar to the concept of visual or interactivity literacy [27].

Our research effort is still at an exploratory stage. Further work is needed to detail impressions and social relations within the user media experience. In addition, ontologies should be formalized by means of the software environments already available. In the near future we shall also test the potentialities of the model for semiotic indexing of hypermedia objects. We believe that our results are promising towards a more comprehensive conceptual model of hypermedia contents.

\section{References}

1. Wright, P., Wallace, J., McCarthy, J.: Aesthetic and Experience-Centered Design. ACM Transactions on Computer-Human Interaction 15(4), Article 18 (2008)

2. Kazmierczak, E.: Design as meaning making: from making things to the design of thinking. Design Studies 19(2), 45-59 (2003)

3. Crilly, N., Maier, A., Clarkson, P.J.: Representing Artifacts as Media: Modelling the Relationship between Designer Intent and Consumer Experience. Journal of Design 2(3), 15-27 (2008)

4. Desmet, P., Hekkert, P.: Framework of product experience. International Journal of Design (1), 57-66 (2007) 
5. Hardman, L., Bulterman, D.C.A., van Rossum, G.: The Amsterdam Hypermedia Model: adding time and context to the Dexter model. Communications of the ACM 37(2), 50-62 (1994)

6. Montero, S., Diaz, P., Aedo, I.: A framework for the analysis and comparison of hypermedia design methods. In: Proc. Applied Informatics 2003, pp. 1053-1058 (2003)

7. Sieckenius De Souza, C.: The Semiotic Engineering of Human-Computer Interaction. The MIT Press, Cambridge (2004)

8. Gangemi, A., Borgo, S., Catenacci, C., Lehmann, J.: Metokis: Task taxonomies for knowledge content. Deliverable D07, Laboratory of Applied Ontology, IRST-CNR (Italy), 80-84 (2005)

9. Toppano, E., Roberto, V.: Semiotic Design and Analysis of Hypermedia. In: Proc. 20th ACM Conference on Hypertext and Hypermedia, HT 2009, Turin, Italy, pp. 367-368 (2009)

10. Benitez, A.B., Martinez, J.M., Rising, H., Salembier, P.: Description of a single multimedia document. In: Manjunath, B.S., Salembier, P., Sikora, T. (eds.) Introduction to MPEG 7: Multimedia Content Description Language, pp. 111-138. Wiley (2002)

11. Martinez, J.M.: MPEG-7 Overview. ISO/IEC JTC1/SC29/WG11 N6828, Palma de Mallorca, Spain (2004)

12. Martinez, J.M., Koenen, R., Pereira, F.: MPEG-7: the generic Multimedia Content Description Standard. Part 1. IEEE Multimedia, 78-87 (2002)

13. Martinez, J.M., Koenen, R., Pereira, F.: MPEG-7: overview of MPEG-7 Description Tools. Part 2. IEEE Multimedia, 83-93 (July-September 2002)

14. Hunter, J.: Adding Multimedia to the Semantic Web - Building an MPEG-7 Ontology. In: Stamou, G., Kollias, S. (eds.) Multimedia Content and the Semantic Web, ch. 3. Wiley (2005)

15. Peirce, C.S.: Collected Writings. In: Hartshorne, C., Weiss, P., Burks, A.W. (eds.), Harvard Univ. Press, Cambridge (1931)

16. Greimas, A.J., Courts, P.: An Analytical Dictionary. Indiana University Press, Bloomington (1982)

17. Lowe, N.J.: A cognitive model. In: Bernstein, M., Gerco, D. (eds.) Reading Hypertext. Eastgate Systems (2009)

18. Kress, G., van Leeuwen, T.: Reading Images. The Grammar of Visual Design, Routledge (2003)

19. Buchanan, R.: Declaration by design: rhetoric, argument, and demonstration in design practice. Design Issues 2(1), 4-22 (1985)

20. Hekkert, P.: Design Aesthetics: principles of pleasure in design. Psychology Science 48(2), 157-172 (2006)

21. Hekkert, P., Leder, H.: Product aesthetics. In: Schifferstein, Hekkert (eds.) Product Experience, ch. 10. Elsevier Science (2007)

22. Blythe, M., Hassenzahl, M.: The semantic of fun: differentiating enjoyable experiences. In: Blythe, M.A., Monk, A.F., Overbeeke, K., Wright, P.C. (eds.) Funology: from Usability to Enjoyment, pp. 91-100. Kluwer Academic Publisher (2003)

23. Demir, E., Desmet, P.M.A., Hekkert, P.: Appraisal patterns of emotions in human-product interaction. International Journal of Design 3(2), 41-51 (2009)

24. Russel, J.A.: A circumplex model of affect. Journal of Personality and Social Psychology 39(6), 1161-1178 (1980)

25. Bartle, R.: Presence and Flow: ill-fitting clothes for virtual worlds. Techne 10(3), Article 4 (2007)

26. Kouprie, M., Visser, F.S.: A framework for empathy in design: stepping into and out of the user' s life. Journal of Engineering Design 20(5), 437-448 (2009)

27. Lim, Y., Lee, S., Kim, D.: Interactivity attributes for expression-oriented Interaction Design. International Journal of Design 5(3), 113-128 (2011) 